\title{
Gravity tests with INPOP planetary ephemerides
}

\author{
A. Fienga ${ }^{1,2}$, J. Laskar ${ }^{1}$, P. Kuchynka ${ }^{1}$, C. Leponcin-Lafitte ${ }^{3}$, \\ H. Manche ${ }^{1}$ and M. Gastineau ${ }^{1}$ \\ ${ }^{1}$ Astronomie et Systèmes Dynamiques, IMCCE-CNRS UMR8028, 77 Av. Denfert-Rochereau, \\ 75014 Paris, France and ${ }^{2}$ Observatoire de Besançon- CNRS UMR6213, 41bis Av. de \\ l'Observatoire, 25000 Besançon and ${ }^{3}$ SYRTE-CNRS UMR8630, Observatoire de Paris, 77 Av. \\ Denfert-Rochereau, France
}

\begin{abstract}
We present here several gravity tests made with the latest INPOP08 planetary ephemerides. We first propose two methods to estimate the PPN parameter $\beta$ and its correlated value, the Sun $J_{2}$, and we discuss the correlation between the Sun $J_{2}$ and the mass of the asteroid ring. We estimate a possible advance in the planet perihelia. We also show that no constant acceleration larger than $1 / 4$ of the Pioneer anomaly is compatible with the observed motion of the planets in our Solar System.
\end{abstract}

\section{Introduction}

Since 1981 and the use in the JPL NASA orbit determination software of the EinsteinHoffman equations of motion in the frame of the PPN formulism (Will 1971, Moyer 1981), deviation of the gravity from the General Relativity (GR) theory can be measured by the estimation of parameters scaling the gravity to the GR, essentially $\gamma, \beta$. The most precise measurement of $\gamma$ can be obtained in experiments of light deflection by the Sun or a major planet and gravitational time delay experiments (Kopeikin and Makarov 2008, Bertotti et al. 2003). However $\beta$ can only be estimated in using the advance of the planet perihelion in association with a $\gamma$ determination (Will 2006, Williams et al. 2009).

At the same time, the planetary ephemerides have made a huge improvement in modeling of solar system bodies, and the analysis of tracking observations of spacecraft became a powerful tool to constrain the dynamics of these bodies and to determine the PPN parameters such as $\gamma$ and $\beta$ (Pitjeva 1986).

Besides the estimations of parameters such as $\operatorname{PPN} \beta$, the planetary ephemerides are an interesting tool to study the impact of the Pioneer anomaly (PA) in the solar system. Since the unexplained acceleration exhibited by the two Pioneer spacecrafts, sent more than 30 years ago to the limit of our solar system, was confirmed by Anderson et al. (2002), many possible explanations were proposed and investigated. Some of the proposed explanations can be tested thanks to planetary ephemerides. Finally as GR was the explanation of the secular advance of the perihelion of Mercury detected by the old optical observations, one can be interested in using new accurate radar tracking data to detect supplementary advances unexplained by GR (Pitjeva 1986).

In this paper, we give several estimations of $\operatorname{PPN} \beta$, as well as the Sun oblateness coefficient $J_{2}$. We show how the planetary ephemerides can help to solve the Pioneer anomaly, and we estimate the limit of detection of possible extra advances in perihelia based on modern observations of planets. 
Table 1. The first 2 columns give the a-priori INPOP uncertainties in geocentric angles and distances limited by the observation accuracy. In the third column are the estimates of the general relativity and Sun oblateness contribution to the perihelion rate $\dot{\omega}$, of Mercury, Venus and Mars. Column 4 gives the $\mathrm{S} / \mathrm{N}$ ratio estimated over the time period of column 5.

\begin{tabular}{|c|c|c|c|c|c|c|c|c|c|c|c|}
\hline Planets & $\begin{array}{c}\text { INPOP } \\
\text { angle }\end{array}$ & $\begin{array}{l}\text { accuracy } \\
\text { distance }\end{array}$ & $" \begin{array}{c}\dot{\omega} \\
\end{array}$ & $\mathrm{S} / \mathrm{N}$ & $\begin{array}{l}\text { period } \\
\text { years }\end{array}$ & Planets & $\begin{array}{c}\text { INPOP } \\
\text { angle }\end{array}$ & $\begin{array}{l}\text { accuracy } \\
\text { distance }\end{array}$ & $\begin{array}{r}\dot{\omega} \\
" / \mathrm{yr}\end{array}$ & $\mathrm{S} / \mathrm{N}$ & $\begin{array}{l}\text { period } \\
\text { year }\end{array}$ \\
\hline Venus & $0.001 "$ & $4 \mathrm{~m}$ & 0.086 & $\begin{array}{l}172 \\
344\end{array}$ & $\begin{array}{l}2 \\
4\end{array}$ & Mars & $0.001 "$ & $2 \mathrm{~m}$ & 0.013 & $\begin{array}{l}130 \\
390\end{array}$ & $\begin{array}{l}10 \\
30\end{array}$ \\
\hline Mercury & $0.050 "$ & $1 \mathrm{~km}$ & 0.43 & 300 & 35 & & & & & & \\
\hline
\end{tabular}

\section{Determination of PPN $\beta$ and the Sun oblateness $J_{2}$}

\subsection{Planetary ephemerides accuracy}

Thanks to the high precision achieved with the observations deduced from spacecraft tracking, it becomes possible to estimate relativistic parameters $\gamma$ and $\beta$ of the Parametrized Post-Newtonian formalism of General Relativity (Will, 1993). Nevertheless, if $\gamma$ plays a role in the equations of motion, it is worth noting that light propagation is only sensitive to that parameter. PPN $\gamma$ can then be estimated with high accuracy by light deflection measurements by VLBI (Shapiro et al. 2004, Lambert \& Le PoncinLafitte, 2009), by time delay during an interplanetary roundtrip, and by Doppler tracking data of a space mission (see for instance the Cassini experiment, Bertotti et al. 2003). This is also why, in the following, we assume $\gamma=1$ in order to test only the sensitivity of PPN $\beta$ on the perihelion's advance of planets. However, the Sun oblateness $J_{2}$ plays also a key role in this phenomena. Indeed, the usual expression of the advance of perihelion is given by (Will 2006)

$$
\Delta \omega=\frac{2 \varpi(2 \gamma-\beta+2) G M_{\mathrm{sun}}}{a\left(1-e^{2}\right) c^{2}}+\frac{3 \varpi J_{2} R_{\mathrm{sun}}^{2}}{a^{2}\left(1-e^{2}\right)^{2}}
$$

where $G$ and $c$ are the newtonian gravitational constant and the speed of light in vacuum, respectively. $J_{2}, M_{\text {sun }}$ and $R_{\text {sun }}$ are the Sun oblateness, mass and equatorial radius, respectively, while $a$ and $e$ are the semi-major axis and the eccentricity of the precessing planet. The PPN $\beta$ is, thus, correlated with the Sun oblateness $J_{2}$ through this linear relation. But, the $\beta$ coefficient varies as $1 / a$, while the $J_{2}$ coefficient is proportional to $1 / a^{2}$. Using data from different planets will, thus, allow to decorrelate these two parameters. MEX and VEX tracking data have actually led to an important improvement of Mars and Venus orbits in INPOP08 (Fienga et al. 2009).

Indeed, we can evaluate the impact of the observations of a specific planet on the determination of $J_{2}$ and $\beta$ by dividing the advance of the perihelion over the time span of observations by the angle uncertainty of INPOP (table 1). For the same observational accuracy, it appears that Venus data are seven times more efficient than Mars to test general relativity and to estimate the Sun $J_{2}$. Therefore, if the VEX mission is extended from 2 years to 4 years, and if VLBI observations of the spacecraft are done with an accuracy of about 1 mas, VEX data will be as important for the PPN and Sun $J_{2}$ estimations as the direct 800-meter accuracy radar ranging on Mercury. Besides, the Mars data are still very important because of the long time span of observations of very good quality obtained since the Viking mission in 1978.

Thanks to the information brought by the combination of very accurate tracking data of spacecraft orbiting different planets, the planetary ephemerides become thus an interesting tool for gravity testing. In the following, we give some examples of such tests. 

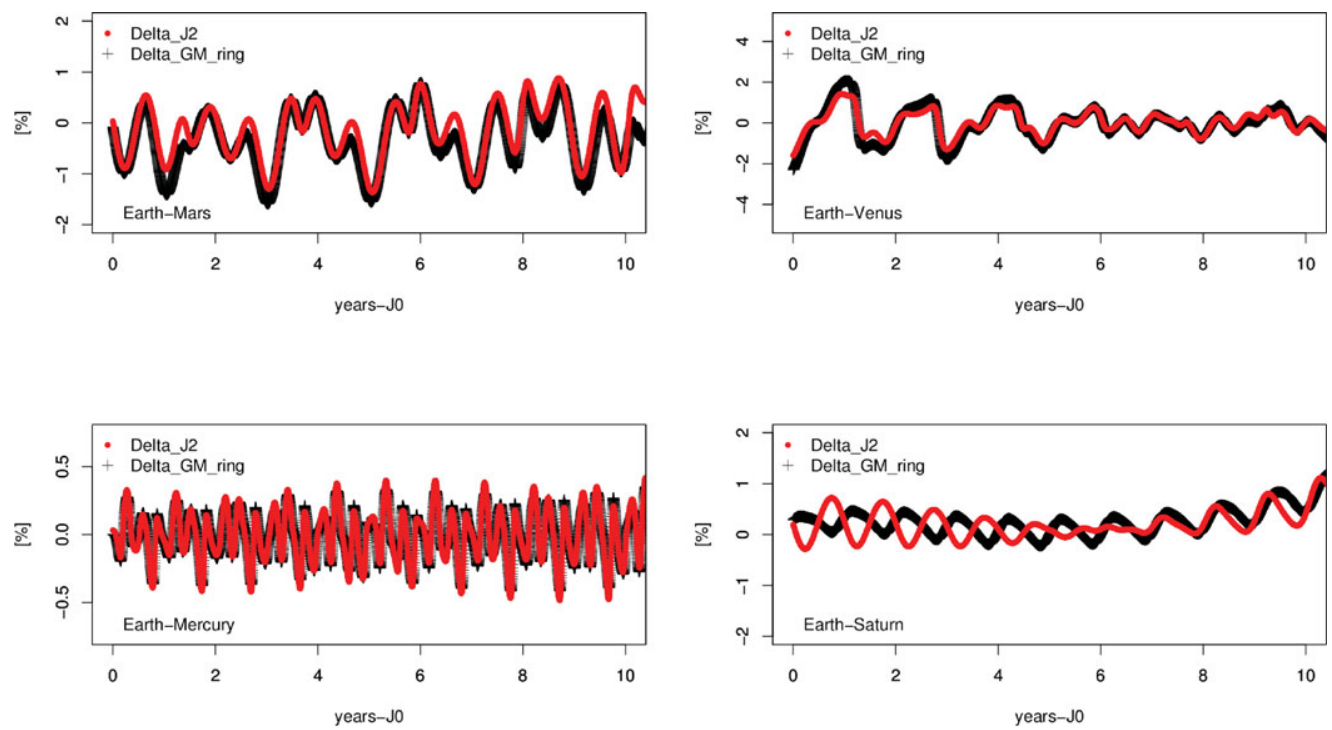

Figure 1. Residuals obtained by comparisons between Mercury direct range, MGS/MO, MEX, VEX and Cassini range tracking data and ephemerides perturbed by a small change in the Sun $J_{2}(12 \%)$ and by a small change in the mass of the asteroid ring (17\%).

\subsection{Correlation between the Sun $J_{2}$ and asteroid modeling}

The advance of the perihelion induced by general relativity and the Sun $J_{2}$ has an impact very similar to the advance induced by the main-belt asteroids on the inner planet orbits. In INPOP08, a ring was added to average the perturbations induced by the main-belt asteroids which cannot be fitted individually by tracking observations. This ring has its physical characteristics (mass and distance to the Sun) estimated independently from the fit by considering the albedos and physical properties of 24635 asteroids (for more details see Kuchynka et al. 2008).

As illustrated in figure 1, there is a correlation between the effect on the geocentric distance of the modeling of the ring as done in INPOP08, in one hand, and the effect of the Sun oblatness in the other hand. Indeed, on these plots, one may see how a small change in the value of the Sun $J_{2}(12 \%)$ induces, after the refit of the planet initial conditions a periodic effect very similar in amplitude and frequency as a change in the mass of the asteroid ring (17\%). This effect is obvious on Mercury, Mars and Venus distances to the Earth, but not for Saturn. The Saturn-Earth distances are indeed not affected in the same way. We can also conclude that, when new accurate observations of outer planets will be obtained, they will be very useful to decorralate asteroid effects on planet orbits by combination with inner planet data. Finally, it stresses the crucial importance of having a model of the asteroid perturbations as a fixed ring, characterized independantly from the fit of planetary ephemerides.

By fixing the ring, we limit then an overestimation of the value of the Sun $J_{2}$ merging in this value some effects induced by the asteroids.

Two different but complementary analysis and determination of PPN $\beta$ and the Sun $J_{2}$ are presented in the next sections with a fixed model of asteroid perturbations (same values of asteroid and ring masses and of densities as INPOP08). 


\subsection{Estimations by least squares}

The first approach is based on the classic least square estimation of parameters during the fit of planetary equations of motion to observations. To check numerically the simplified assertion made in the introduction (section 1), we estimate here what is the impact of each data set in the determination of $J_{2}$ and $\beta$ : several adjustments of the initial conditions of planets and the parameters $J_{2}$ and $\beta$ are made using different sets of observations. This leads to 32 adjustments based on INPOP08. For each fit, changes were made in the selection of Mars and Venus data in order to estimate the impact of each important set of observations in the fit of the Sun $J_{2}$ and PPN $\beta$. We look at the variations in the estimation errors of the 2 parameters and we use the $1-\sigma$ given by the least squares as an indicator of this uncertainty. With this method, we are able to quantify the influence of each data set on the determination of the pair $\left(\beta, J_{2}\right)$ as well as the stability of the determinations of the parameters. Indeed, these variations in the error's estimation of the pair $\left(\beta, J_{2}\right)$ are a relevant indicator of the uncertainty of the fit of $\beta$ and $J_{2}$.

To take into account the correlation between $J_{2}$ and $\beta$, we use two modes of adjustments: in mode1, $\beta$ or $J_{2}$ are fitted alone with the initial conditions of planets; in mode2, both parameters are fitted simultaneously with the initial conditions of planets.

The results are summarized in table 2 . As expected from the correlation of $J_{2}$ and $\beta$, the determinations of the Sun $J_{2}$ and $\beta$ made separately (i.e. mode1) give better $\sigma$ than fits including the simultaneous $\left(\beta, J_{2}\right)$ determination (mode2). The best results for a correlated determination of $J_{2}$ and $\beta$ (mode2) are obtained when only the most accurate observations of Mars (MGS/MO, MEX and Viking) and Venus (VEX) are used simultaneously.

Moreover, we note that the combined use of Venus ranging data and the complete data set for Mars does not really improve the separated determination (mode1) of $\beta$ and $J_{2}$, mainly due to the low accuracy of these observations, but a contrario, it gives better correlated estimations (mode2). This is also consistent with the fact that fitting over observations from two different planets helps to decorrelate $J_{2}$ and $\beta$. Furthermore, the Viking data, by prolonging the fit interval with observations of rather good accuracies, allow a decreasing of the uncertainties of about $20 \%$ for $J_{2}$ and about $10 \%$ for $\beta$. Finally, it appears that VEX data improve the determinations in a significant way: decreasing by $31 \%$ the least squares $\sigma$ of the $J_{2}$ estimation and $48 \%$ for $\beta$. Less than 2 years of VEX data have a bigger influence than a large interval (more than 30 years) of accurate Mars observations. This is especially relevant for the PPN parameter $\beta$ with an improvement of about $48 \%$ of the accuracy between a determination including only Mars data and another one with both Mars and VEX data. In the same time, the improvement induced by the addition of Viking data is about $20 \%$ for $J_{2}$ and $10 \%$ for the PPN parameter $\beta$. These figures show the crucial role of the VEX data before the use of future data from the ongoing generation of Mercury orbiters.

\subsection{Incremental method and sensitivity estimation}

An alternate strategy to study the sensitivity of the planetary ephemerides to $J_{2}$ and PPN $\beta$ is to estimate how does an ephemeris built using different values for $J_{2}$ and PPN $\beta$ and fitted on the same set of observations as INPOP08 differ from INPOP08. Such differences give an indication on how observations are sensitive to these parameters and with which accuracy we can estimate a parameter such as $\beta$.

To test such sensitivity, we focus our attention on the postfit residuals of the most accurate data sets used in the INPOP08 adjustment: the Mercury direct range, because of its sensitivity to general relativity and to the Sun $J_{2}$; VEX, MEX and MGS/MO data, because of their high accuracy and simulated $S / N$ presented on table 1 ; and Jupiter 
Table 2. 1- $\sigma$ least squares obtained for $J_{2}$ and $\beta$ using several sets of observations.

\begin{tabular}{|c|c|c|c|c|c|c|c|}
\hline & mode & $\begin{array}{l}J_{2} \\
\times 10^{7}\end{array}$ & $\begin{array}{l}(1-\beta) \\
\times 10^{3}\end{array}$ & & mode & $\begin{array}{l}J_{2} \\
\times 10^{7}\end{array}$ & $\begin{array}{l}(1-\beta) \\
\times 10^{3}\end{array}$ \\
\hline Modern Mars & 1 & 0.181 & & Impact of VEX & 1 & 0.144 & \\
\hline \multirow{2}{*}{$\mathrm{MEX}+\mathrm{MGS} / \mathrm{MO}$} & 1 & & 0.042 & Mars + VEX & 1 & & 0.025 \\
\hline & 2 & 0.367 & 0.085 & & 2 & 0.208 & 0.037 \\
\hline Impact of $\mathrm{Vkg}$ & 1 & 0.161 & & Impact of old Venus & 1 & 0.188 & \\
\hline $\mathrm{MEX}+\mathrm{MGS} / \mathrm{MO}+\mathrm{Vkg}$ & 1 & & 0.040 & Mars + old Venus & 1 & & 0.040 \\
\hline$=$ Mars & 2 & 0.302 & 0.076 & & 2 & 0.283 & 0.060 \\
\hline
\end{tabular}

Galileo data and Saturn Cassini normal points. These 2 latest data sets are selected because they induce a global improvement of the planetary ephemerides and especially of the Earth orbit.

To estimate the sensitivity of these 7 most accurate sets of data used in the INPOP08 adjustment to the variations of values of $J_{2}$ and $\operatorname{PPN} \beta$, we have estimated and plotted the $S / N$ ratio defined as:

$$
S / N=\frac{\sigma_{i, j}-\sigma_{0,0}}{\sigma_{0,0}}
$$

where $\sigma_{i, j}$ is the 1-sigma dispersion of the postfit residuals of an ephemeris based on INPOP08 but with values of $J_{2}$ and PPN $\beta$ different from the ones used in INPOP08 (which are $\beta=1.0$ and $J_{2}=1.82 \times 10^{-7}$ ) and fitted to all the INPOP08 data sets, and $\sigma_{0,0}$ is the 1-sigma dispersion of the postfit INPOP08 residuals. We have used 9 values of $J_{2}$ varying from $1.45 \times 10^{-7}$ to $3.05 \times 10^{-7}$ with a 0.2 step, and 24 values of PPN $\beta$, building then 192 different ephemerides. The 24 values of $\beta$ are distributed over 2 windows: a global one based on 12 values of $\beta$ varying from 0.997 to 1.003 with a 0.0005 step (window 1) and from 0.9996 to 1.0004 with a step of 0.0001 (window 2). Results presented as the $S / N$ percentage, are plotted in figure 2 . As one can see in figure 2 , the impact of the PPN $\beta$ is not symmetric with respect to $\beta=1$. In figure 2 , one notices also the direct correlation between the $S / N$ obtained with MGS/MO and MEX data and the one obtained for VEX.

One may see in figure 2 that the $S / N$ of the Jupiter and Saturn data sets are sensitive to changes in $J_{2}$ and PPN $\beta$. The sensitivity of these data sets are not crucial for the analysis, but they reflect the impact of the use of such observations in the improvement of the Earth orbit and then the sensitivity of the Earth orbit to the gravity testing. In table 3, we have gathered minimum and maximum values of PPN $\beta$ defining the sensitivity interval of the different data sets. The sensitivity interval is the interval of PPN $\beta$ for which the $S / N$ remains below $5 \%$. Values of PPN $\beta$ greater than the maximum value given in table 3 , or smaller than the minimum value, cannot be seen as realistic in comparison to modern observations. By considering figure 2 and table 3 it appears that MGS/MO and MEX data provide the most narrow interval of sensitivity with $0.99995<\beta<1.0002$. This interval is in agreement with the latest determinations done by Williams et al. (2009), Fienga et al. (2008) and Pitjeva (2006).

\section{Secular advances of planetary perihelia}

We are interested here in evaluating if the observations used to fit INPOP08 would be sensitive to supplementary precessions of the planet orbits. Such anomalous precessions that would be unexplained by general relativity have been recently investigated (Iorio, 

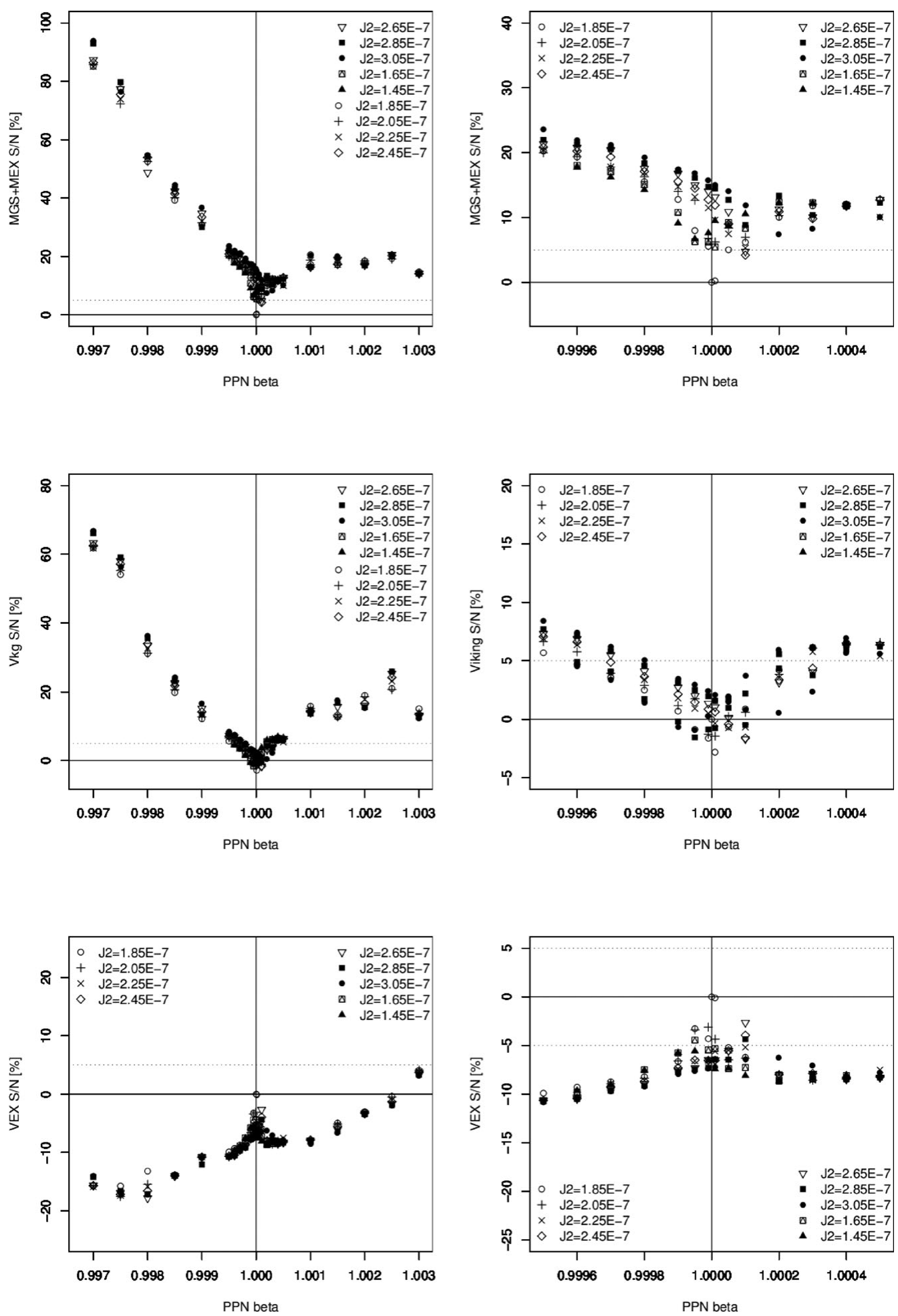

Figure 2. Residuals obtained by comparisons between observations and ephemerides estimated with different values of PPN $\beta$ (values given on x-axis of each subframes) and different values of the Sun $J_{2}$. 

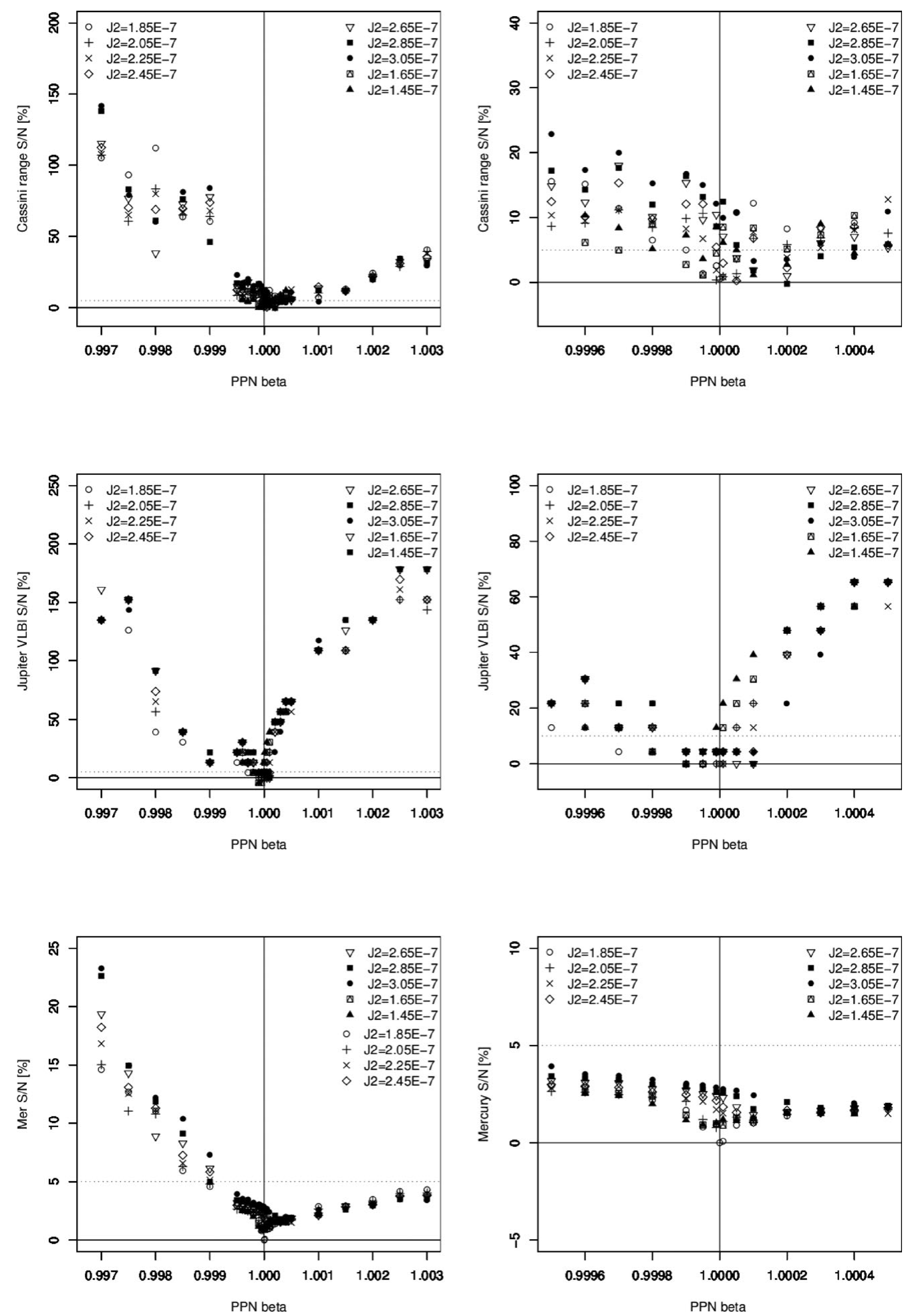

Figure 3. Residuals obtained by comparisons between Mercury, Jupiter VLBI and Saturn range observations and ephemerides estimated with different values of PPN $\beta$ (values given on x-axis of each subframes) and different values of the Sun $J_{2}$. 
Table 3. $\beta$ intervals in which the residuals stay below the $5 \%$ limit. The values of $\beta$ given here are estimated for $\gamma=1$.

\begin{tabular}{|c|c|c|c|c|c|}
\hline Data & $\beta \min$ & $\beta \max \mid$ Data & $\beta \min$ & $\beta \max \mid$ Data & $\beta \min \beta \max$ \\
\hline $\mathrm{MGS} / \mathrm{MO}+\mathrm{MEX}$ & 0.99995 & $1.0002 \mid$ Jupiter VLBI & 0.9996 & $1.0002 \mid$ Viking & 0.99951 .0002 \\
\hline VEX & 0.99990 & 1.0002 Saturn Cassini range & 0.9998 & 1.0005 Mercury & 0.99851 .005 \\
\hline
\end{tabular}

2009). To estimate the sensitivity of the modern tracking data, we first fix $J_{2}=1.8 \times 10^{-7}$, $\beta=1$ and $\gamma=1$. By fixing the value of the Sun $J_{2}$, we then isolated the impact of the secular advance of the perihelion, $\dot{\varpi}_{\text {sup }}$, for one given value of $J_{2}$.

For each different value of $\dot{\varpi}_{\text {sup }}$, initial conditions of planets are fit to the INPOP08 observations and we compare the postfit residuals to the INPOP08 ones. We focused our study on the same sets of observations as for the $J_{2}, \beta$ study. As one can see in figure 4 , the behaviour of the obtained $\mathrm{S} / \mathrm{N}$ (as defined in section 2.4) is symmetrical to a minimum value, this minimal value being centered around $\dot{\varpi}_{\text {sup }}=0$ or not. This symmetry explains why in table 4 we give an interval of $\dot{\varpi}_{\text {sup }}$ for which the minimum of $\mathrm{S} / \mathrm{N}$ is obtained. One can then compare these values to those published by (Pitjeva 2009 ). For all the planets, except Saturn, the values of $\dot{\varpi}_{\text {sup }}$ minimizing the residuals are not significantly different from zero. One can note that the best constraint on the Earth orbit is given by the Jupiter VLBI data set which gives the narrowest interval of $\dot{\varpi}_{\text {sup }}$. For Saturn, an offset in the minimum of the $\mathrm{S} / \mathrm{N}$ is obtained for the Cassini tracking data set $(-10 \pm 8)$ and the VEX data set $(200 \pm 160)$. These estimations lead to determinations of a supplementary precession of the Saturn orbit that are only marginally statistically significant. By comparisons, (Pitjeva 2009) the value is very close to the one we obtain by considering only the $\mathrm{S} / \mathrm{N}$ induced on the Cassini observations. This result shows how important the description of the method used for evaluating such quantities.

To test the stability of the estimations and as it is well-known that the asteroids induce a global precession of the inner planets perihelia, we operate the same computations with small changes in the mass of the ring $(20 \%)$ and in the Sun $J_{2}(5 \%)$ values. The obtained variations of the $\mathrm{S} / \mathrm{N}$ are plotted in figure 4 where the dash curves are the results obtained with the change in the mass of the ring and the longdash curves are the ones deduced from the $J_{2}$. Some changes are noticeable for Viking and Jupiter, however, for Cassini and VEX, the minimum are stable.

The investigation about a statistically significant advance in the Saturn perihelion has to be continued in using more Cassini and VEX data. Indeed, a prolongation of the interval of time covered by these two data sets will improve the accuracy of the estimations.

\section{Does the Pioneer anomaly impact the ephemerides?}

Since 2002 and the confirmation by several teams of the detection of acceleration anomalies in the tracking of several spacecrafts, three features of possible explanations can be given; first, the detected acceleration is not really an acceleration but is a manifestation of a mis-modeling in the Doppler and ranging signals taped by navigation teams. Second, the anomaly is a mis-modeling in the orbit of the probe itself induced by a technical problem or misunderstandings of the spacecraft techniques. The third cause invocated is a generalization of the second one by implying a mis-modeling in the dynamics of the probe but also of all objects in the solar system and beyond. Thus, if the equivalence 
Table 4. $\dot{\varpi}_{\text {sup }}$ intervals minimizing postfit residuals.

\begin{tabular}{|c|c|c|c|c|c|c|c|c|}
\hline \multirow[b]{2}{*}{ Data } & \multicolumn{8}{|c|}{$\dot{\varpi}_{\text {sup }}[\mathrm{mas} / \mathrm{cy}]$} \\
\hline & Mer & Ven & EMB & Mars & Jup & Saturn & $\begin{array}{l}\text { Ura } \\
\times 10^{-4}\end{array}$ & $\begin{array}{l}\text { Nep } \\
\times 10^{-4}\end{array}$ \\
\hline Mercury & $-10 \pm 30$ & $30 \pm 130$ & $0 \pm 40$ & $>2000$ & $>2000$ & $0 \pm 200$ & $>20$ & $>20$ \\
\hline VEX & $0 \pm 200$ & $18 \pm 22$ & $0 \pm 4$ & $0 \pm 1.4$ & $0 \pm 200$ & $200 \pm 160$ & $0 \pm 2$ & $>20$ \\
\hline MGS/MO+MEX & $0 \pm 200$ & $-24 \pm 34$ & $-0.4 \pm 0.8$ & $0.4 \pm 0.6$ & $-20 \pm 180$ & $0 \pm 60$ & $0 \pm 2$ & $0 \pm 10$ \\
\hline Viking & $0 \pm 200$ & $-24 \pm 34$ & $0 \pm 0.2$ & $0 \pm 0.2$ & $-200 \pm 200$ & $0 \pm 10$ & $(4 \pm 4)$ & $0 \pm 10$ \\
\hline Jupiter VLBI & $0 \pm 400$ & $-4 \pm 6$ & $0 \pm 0.016$ & $0 \pm 0.6$ & $142 \pm 156$ & $0 \pm 10$ & $0 \pm 2$ & $0 \pm 2$ \\
\hline $\begin{array}{l}\text { Saturn range } \\
\text { Cassini }\end{array}$ & $>2000$ & $0 \pm 10$ & $0.1 \pm 0.1$ & $0 \pm 0.2$ & $0 \pm 400$ & $-10 \pm 8$ & $0 \pm 2$ & $0 \pm 2$ \\
\hline
\end{tabular}
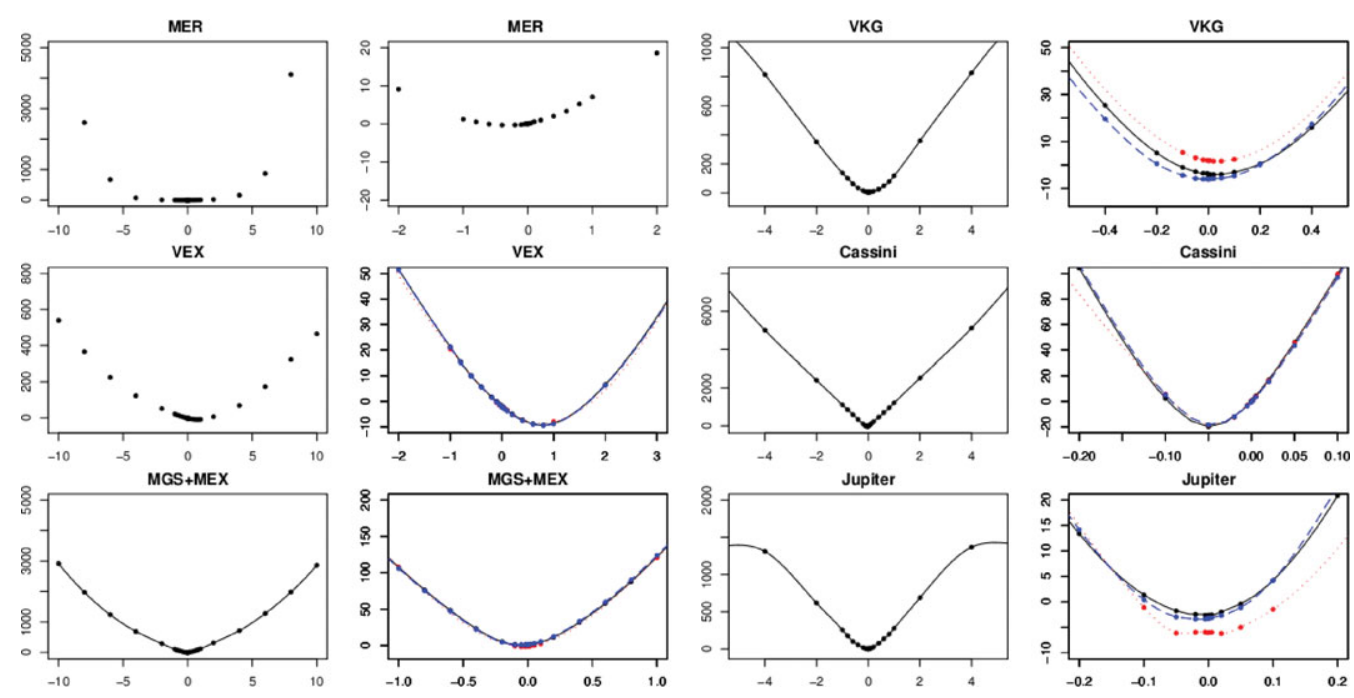

Figure 4. Residuals obtained by comparisons between observations and ephemerides estimated with different values of $\dot{\varpi}_{\text {sup }}$. The dash curves represent residuals obtained with a $20 \%$ change in the mass of the asteroid ring whereas the longdash curses represent residuals obtained with a $5 \%$ change in the J2. The $\mathrm{x}$-axis give the values of $\dot{\varpi}_{\text {sup }} \times 10^{8}$ used in the simulations and the $\mathrm{y}$-axis give the variations of the $\mathrm{S} / \mathrm{N}$ in $\%$.

principal is followed, the equations of motion of the major planets of our solar system have also to be modified in the same manner as the spacecraft dynamical equations are.

We investigate this question by using the INPOP08 planetary ephemerides as a test bed for some hypothesis describing the pioneer anomalies.

A classic description of the pioneer anomalies (PA) is the appearance of a constant acceleration of about $8.75 \times 10^{-10} \mathrm{~m} \mathrm{~s}^{-2}$, Sun-oriented after 20 AU (Anderson et al. 2002). We, thus, add this constant acceleration in the equations of motions of Uranus, Neptune and Pluto.

We have then fit the modified ephemerides to observations usually used to built INPOP08. Residuals obtained after the fit are plotted in Figure 5. The value of the acceleration was changed in a way to obtain a minimum value for which the effect induced by such acceleration becomes detectable in the residuals. As it appears clearly in the residuals of Uranus right ascension, a constant acceleration of $8 \times 10^{-10} \mathrm{~m} \mathrm{~s}^{-2}$ added to 

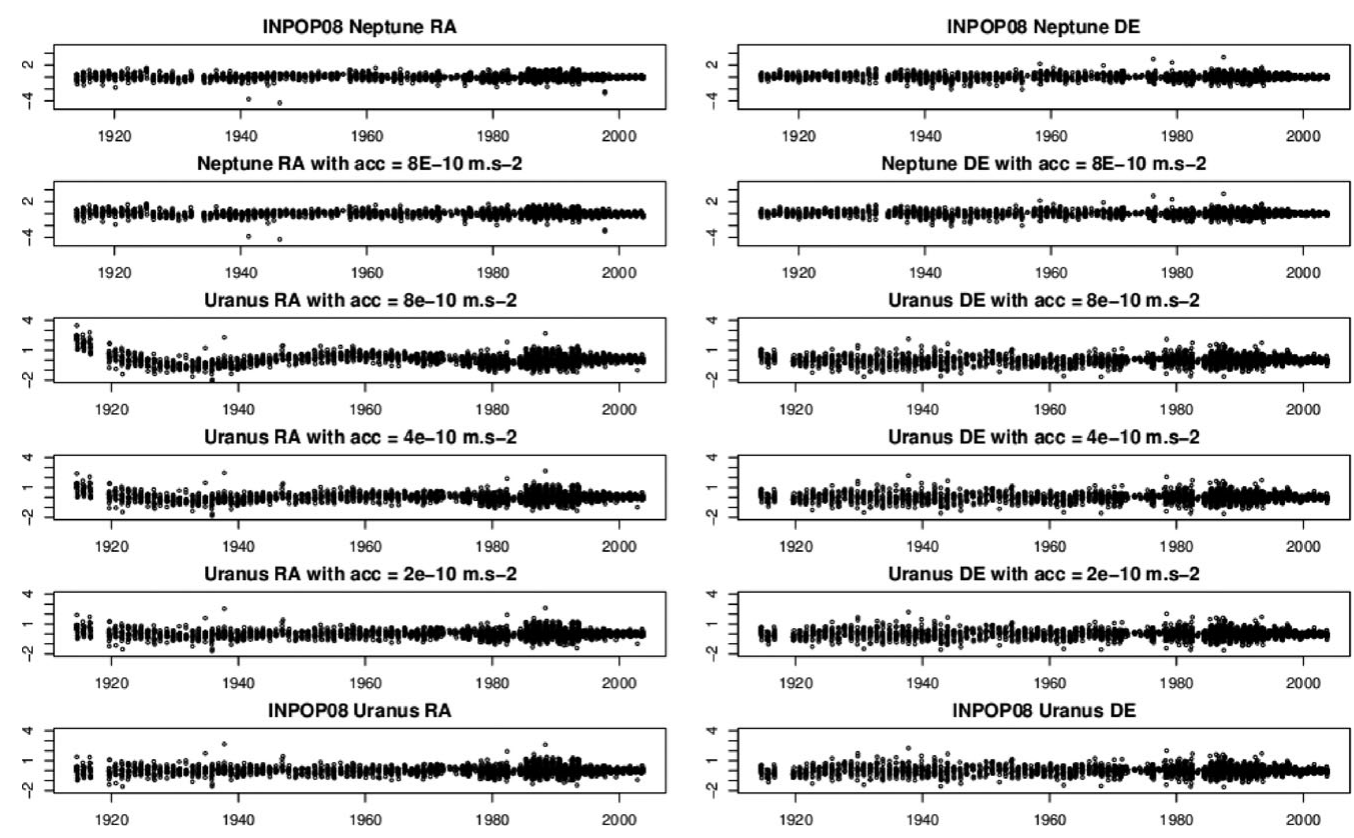

Figure 5. Residuals in right ascension and declination of Neptune and Uranus obtained with INPOP08 (solution of reference) and fitted ephemerides including PA of different magnitudes: from 8 to $2 \times 10^{-10} \mathrm{~m} \mathrm{~s}^{-2}$. The $\mathrm{x}$-axis are years and $\mathrm{y}$-axis is in arcseconds.

the classical Einstein-Hoffmann equations of motion can not be missed, even after the fit of the Uranus initial conditions. A systematic effect remains especially after 1930. This effect cannot be absorbed by the fit or by the noise of the old Uranus observations. By changing the value of the acceleration, one can see that the acceleration must be at least 4 times smaller than the one commonly adopted to be absorbed by the residuals. For Neptune and Pluto, the situation is different. For these planets, the effect of a constant acceleration is absorbed by the fit, as one can see on figure 5 with the postfit and prefit residuals of Neptune.

\section{Conclusions}

Concerning the determination of the PPN parameter $\beta$, an estimation of the sensitivity of planetary ephemerides to this parameter is done following two methods. Our results show that a global fit is needed in order to decorrelate parameters such as PPN $\beta$, the Sun $J_{2}$ and the asteroid pertubations.

We have tested possible detection of an anomalous advance of perihelia of planets. More investigations are needed for the analysis of the perihelion rate of Saturn and more observations of Cassini and VEX data are necessary.

Finally, the results obtained here for the Pioneer Anomaly conclude that no constant acceleration larger than 1/4 the PA can affect the planets of our solar system. If it was so, it would have been detected sooner. In the frame of the equivalence principle, this means that no constant acceleration larger than $1 / 4$ the PA can be realistic. 


\section{References}

Anderson, J. D., Laing, P. A., Lau, E. L., Liu, A. S., Nieto, M. M., \& Turyshev, S. G., 2002, Phys. Rev. D, 65, 082004

Bertotti, B., Iess, L., \& Tortora, P., 2003, Nature, 425, 374

Fienga, A., Manche, H., Laskar, J., \& Gastineau, M., 2009, JOURNEES-2008/ Astrometry, Geodynamics and Astronomical Reference Systems

Fienga, A., Manche, H., Laskar, J., \& Gastineau, M., 2008, A\& A, 477, 315

Iorio, L., 2009, AJ, 137, 3615

Kopeikin, S. \& Makarov, V., 2008, IAU Symposium, 248, 391

Lambert, S. \& Le Poncin-Lafitte, C., 2009, A\&A, 499, 331

Moyer, T. D., 1981, Cel. Mech., 23, 33.

Kuchynka, P., Laskar, J., Fienga, A., Manche, H., \& Somenzi, L., 2009, JOURNEES-2008/ Astrometry, Geodynamics and Astronomical Reference Systems

Pitjeva, E. V., 1986, Byull. Inst. T. A., Ross. Akad. Nauk., 15, 538

Pitjeva, E. V., 2009, JOURNEES-2008/ Astrometry, Geodynamics and Astronomical Reference Systems

Shapiro, S. S., Davis, J. L., Lebach, D. E., \& Gregory, J. S., 2004, Phys. Rev. Lett., 92, 121101

Will, C. M., 2006, Living Rev. Relativity, 9, 3

Will, C. M., 1993, Cambridge University Press, New York, U.S.A.2nd edition

Will, C. M., 1971, Astrophys. J., 163, 611

Williams, J. G., Turyshev, S. G., \& Boggs, D. H., 2009, Int. Jour. Mod. Phys. D, arXiv, gr-qc0507083v2 